\title{
Maternal and umbilical cord erythrocyte omega- 3 and omega- 6 fatty acids and haemorheology in singleton and twin pregnancies
}

\author{
M McFadyen, J Farquharson, F Cockburn
}

See end of article for authors' affiliations

Correspondence to: Dr Farquharson, Department of Biochemistry, Royal Hospital for Sick Children and Queen Mother's Hospital, Yorkhill NHS Trust, Glasgow G3 8SJ, Scotland, UK James.Farquharson@ btinternet.com

Accepted 28 July 2002

\begin{abstract}
Background: Being devoid of both nuclei and mitochondria, mature human erythrocytes provide an opportunity to study membrane structure and function outwith the restrictions of genetic control. With its unique rapid increase in vascularisation, pregnancy is considered the most opportune period in which to investigate blood rheology.

Methods: Maternal and fetal (cord) bloods were retained at delivery from 32 (25 singleton and seven twin) normal pregnancies at two maternity hospitals in the Glasgow area over a nine month period. Erythrocyte fatty acid compositions were assessed by mass spectroscopy, and corresponding membrane deformabilities measured by ultrafiltration through a membrane of $5 \mu \mathrm{m}$ diameter pore size, to mimic placental microcirculation.

Results: Significant direct correlations (Spearman rank) were found between erythrocyte membrane omega-3 docosahexaenoic acid concentrations and corresponding deformabilities in maternal and cord blood from both singleton and twin pregnancies, whereas greater omega-6 arachidonic acid content was associated with increased maternal membrane rigidity. Membrane concentrations of omega-3 fatty acids only correlated strongly both within and between maternal and cord bloods. Mean cord erythrocyte docosahexaenoic acid concentration was higher than maternal in singletons but lower in twins. When maternal erythrocyte concentrations exceeded about 7\% (of total fatty acids), resistance to erythrocyte flow was virtually eliminated.

Conclusions: It may be that a greater maternal intake of docosahexaenoic acid should be encouraged in some pregnancies for optimal tissue perfusion. Fetal demand for docosahexaenoic acid may not be entirely satisfied in multiple pregnancies.
\end{abstract}

$\mathrm{D}$ uring pregnancy, supply of nutrients to the fetus is dependent on maternal diet and metabolism. Nutritional deficiencies have been highlighted in vegetarian and vegan mothers, and multiple pregnancies place an additional strain on such provision. ${ }^{1}$

The causes of the major complications of pregnancy, namely pregnancy induced hypertension and pre-eclampsia, are not known. Their adverse vascular pathologies and decreasing incidence from northern to southern Europe may have a parallel in the epidemiology of cardiovascular disease and an association with consumption of fish rich in omega-3 fatty acids. $^{2-4}$

Omega-3 (or n-3) long chain polyunsaturated fatty acids (LCPUFAs) have been shown to reduce the incidence of miscarriage in persistent antiphospholipid syndrome. ${ }^{5}$ Amelioration of the condition by low dose aspirin therapy may indicate involvement of fatty acids in inducing antibody production, as salicylate inactivation of cyclo-oxygenases directly inhibits arachidonic acid (AA; C20:4 n-6) metabolism. ${ }^{6}$

In spite of several taskforce reports that have emphasised the maternal (and fetal) dietary need for LCPUFAs, rather than their essential fatty acid precursors, during pregnancy, ${ }^{78}$ it is probably true that the implementation of their recommendations has not been universal. In terms of overall maternal fatty acid requirements it is likely that, as they can be synthesised de novo, neither saturated nor monounsaturated fatty acids will play a pivotal role in that regard. The dietary essential fatty acids, linoleic (Cl8:2 n-6) and $\alpha$-linolenic (C18:3 n-3) cannot be synthesised in humans and are parent molecules of the n-6 and n-3 series LCPUFAs, which are critical to the formation and function of all biological membranes. Deficiencies in n-6 LCPUFAs are rare, as lino- leic acid is abundant in non-restrictive diets. However, because of both low dietary intake and rate of synthetic conversion of $\alpha$-linolenic acid, the major n-3 LCPUFA product, docosahexaenoic acid (DHA; C22:6 n-3), which is prominent in most tissues, can be deficient. ${ }^{10}$ Probably because of its high degree of unsaturation, and resultant helical configuration, DHA is thought to contribute disproportionately to cell surface deformability, a property essential in facilitating transport of erythrocytes of $8 \mu \mathrm{m}$ diameter through the 3-4 $\mu \mathrm{m}$ diameter uteroplacental capillary vasculature. ${ }^{11}$ The corollary to this is the understanding that n-6 LCPUFAs (particularly AA) may exhibit an opposing effect on membranes by increasing membrane rigidity, such that their presence in excess may be detrimental.

We therefore sought to establish reference ranges for both maternal and fetal erythrocyte fatty acid concentrations from normal singleton and twin deliveries. To identify associations between individual fatty acid concentrations and membrane deformabilities, all erythrocyte specimens would be analysed by their passage (as measured by transit times) through a standardised filtration system.

\section{METHODS}

After both maternal consent and ethics committee approval had been granted, 32 mothers who had undergone uncomplicated term singleton $(\mathrm{n}=25)$ and near or at-term twin

Abbreviations: LCPUFA, long chain polyunsaturated fatty acid; $A A$, arachidonic acid; DHA, docosahexaenoic acid; DPA, docosapentaenoic acid 
Table 1 Maternal and infant details and maternal and umbilical cord erythrocyte haematology from normal singleton and twin pregnancies

\begin{tabular}{|c|c|c|}
\hline & $\begin{array}{l}\text { Singletons } \\
(n=25)\end{array}$ & Twins $(n=7)$ \\
\hline Maternal age (years) & $28.9(4.9)$ & $29.7(2.6)$ \\
\hline Gestational age (weeks) & $38.7(1.1)$ & $35.9(1.6)^{*}$ \\
\hline Birth weight (g) & 3278 (439) & $2475(478)$ * \\
\hline \multicolumn{3}{|c|}{ Mean corpuscular volume (fl) } \\
\hline Maternal & $89.5(7.2)$ & $90.4(5.9)$ \\
\hline Infant & $109.9(7.8)$ & $114.7(4.6)^{* *}$ \\
\hline
\end{tabular}

Results are given as mean (SD).

Significant between group (singleton $v$ twin) differences calculated by Student's $t$ test; * $p<0.001,{ }^{* *} p=0.022$

$(\mathrm{n}=7)$ pregnancies were recruited into the study over a continuous period of nine months. Maternal and infant characteristics were obtained from case records.

Within 24 hours before delivery, $10 \mathrm{ml}$ maternal blood was withdrawn from the antecubital vein, placed immediately in a potassium EDTA tube to prevent clotting, and $1 \mathrm{ml}$ retained for estimation of erythrocyte mean corpuscular volume, erythrocyte count, and packed cell volume. The remaining blood was divided: $2 \mathrm{ml}$ was centrifuged (1000 g, $5 \mathrm{~min}$ ), erythrocytes washed twice with isotonic saline, and stored at $-70^{\circ} \mathrm{C}$ before fatty acid analysis. Corresponding venous cord blood, collected immediately after cord clamping and within five minutes of delivery, was similarly prepared. Erythrocytes separated from the final aliquot $(7 \mathrm{ml})$ were passed through a cellulose column to ensure complete removal of white blood cells, and resuspended in a volume of phosphate buffered saline (calculated from the erythrocyte count) to yield $0.52 \times 10^{9}$ erythrocytes $/ \mathrm{ml}$. Erythrocyte deformabilities were measured within five hours of venepuncture by filtration of this cell suspension, at constant pressure, through a $5 \mu \mathrm{m}$ diameter pore size polycarbonate membrane as described. ${ }^{12}$

Erythrocyte membrane total phospholipid fatty acids were extracted, derivatised, and subjected to gas chromatographic/ mass spectroscopic analysis performed as previously outlined. ${ }^{13}$

\section{Statistical analysis}

Between group (singleton $v$ twin) differences in continuous variables were analysed by Student's $t$ test. Associations between erythrocyte (maternal and cord) phospholipid fatty acid concentrations (as wt \% of total fatty acids) and erythrocyte transit times (log transformed) were assessed for both

Table 2 Maternal erythrocyte total phospholipid fatty acid concentrations (wt \% of total fatty acids) and erythrocyte deformabilities (as measured by their transit times in seconds (log transformed; $\log _{10}$ TT) through a membrane of $5 \mu \mathrm{m}$ diameter pore size) from normal singleton and twin pregnancies

\begin{tabular}{|c|c|c|c|c|c|c|}
\hline & \multicolumn{3}{|c|}{ Singletons $(n=25)$} & \multicolumn{3}{|l|}{ Twins $(n=7)$} \\
\hline & Fatty acid & $r$ & $\mathrm{p}$ Value & Fatty acid & $r$ & $p$ Value \\
\hline Palmitic C16:0 & $30.2(1.9)$ & 0.03 & 0.902 & $31.4(2.9)$ & 0.68 & 0.094 \\
\hline Stearic C 18:0 & $13.9(1.2)$ & -0.11 & 0.604 & $13.6(0.6)$ & -0.61 & 0.149 \\
\hline Oleic C18:1 n-9 + n-7 & $17.9(1.1)$ & 0.31 & 0.126 & $17.8(1.0)$ & 0.39 & 0.383 \\
\hline Linoleic C 18:2 n-6 & $12.1(1.3)$ & 0.22 & 0.297 & $11.4(1.2)$ & 0.25 & 0.588 \\
\hline Dihomo- - -linolenic C20:3 n-6 & $1.88(0.65)$ & -0.10 & 0.641 & $1.84(0.73)$ & -0.14 & 0.760 \\
\hline AA C20:4 n-6 & $12.3(1.5)$ & 0.47 & $0.017^{*}$ & $11.5(1.1)$ & 0.14 & 0.760 \\
\hline Docosatetraenoic C22:4 n-6 & $2.72(0.81)$ & 0.34 & 0.098 & $2.53(0.68)$ & 0.14 & 0.760 \\
\hline Docosapentaenoic C22:5n-3 & $2.22(0.80)$ & -0.53 & $0.007^{*}$ & $2.53(1.03)$ & -0.96 & $<0.001$ * \\
\hline DHA C22:6 n-3 & $5.94(1.22)$ & -0.55 & $0.004 *$ & $6.51(1.66)$ & -0.78 & $0.048 *$ \\
\hline AA/DHA (wt \% ratio) & $2.19(0.59)$ & 0.60 & $0.002^{*}$ & $1.88(0.53)$ & 0.46 & 0.299 \\
\hline $\log _{10} \pi T$ & \multicolumn{3}{|c|}{$0.29(-0.18$ to 1.01$)$} & \multicolumn{3}{|c|}{$0.11 \quad(-0.03$ to 0.69$)$} \\
\hline
\end{tabular}

Erythrocyte fatty acid concentrations (as weight \% of total fatty acids) are given as mean (SD) and log transit times (in seconds) as medians (ranges). * Statistically significant Spearman rank correlations $(r)$ between individual erythrocyte fatty acid concentrations and transit times.

AA, Arachidonic acid; DHA, docosahexaenoic acid.

Table 3 Umbilical cord erythrocyte total phospholipid fatty acid concentrations (wt \% of total fatty acids) and erythrocyte deformabilities (as measured by their transit times in seconds (log transformed; $\log _{10}$ TT) through a membrane of $5 \mu \mathrm{m}$ diameter pore size) from normal singleton and twin pregnancies

\begin{tabular}{|c|c|c|c|c|c|c|}
\hline & \multicolumn{3}{|c|}{ Singletons $(n=25)$} & \multicolumn{3}{|c|}{ Twins $(n=14)$} \\
\hline & Fatty acid & $r$ & $\mathrm{p}$ Value & Fatty acid & $r$ & $p$ Value \\
\hline Palmitic C16:0 & $31.3(2.1)$ & 0.28 & 0.177 & $33.3(2.7)$ & 0.10 & 0.736 \\
\hline Stearic C18:0 & $16.5(1.3)$ & -0.11 & 0.614 & $15.9(0.9)$ & -0.33 & 0.254 \\
\hline Oleic C18:1 n-9 + n-7 & $15.1(1.4)$ & 0.10 & 0.641 & $15.6(1.0)$ & 0.49 & 0.078 \\
\hline Linoleic C1 8:2 n-6 & $5.00(0.85)$ & 0.09 & 0.683 & $5.28(1.18)$ & -0.26 & 0.362 \\
\hline Dihomo- $\gamma$-linolenic C20:3 n-6 & $3.06(0.78)$ & -0.36 & 0.077 & $2.91(0.87)$ & 0.00 & 0.998 \\
\hline AA C20:4 n-6 & $17.0(1.3)$ & 0.31 & 0.137 & $16.5(1.5)$ & 0.46 & 0.102 \\
\hline Docosatetraenoic C22:4 n-6 & $3.56(0.50)$ & 0.29 & 0.160 & $2.96(0.90)$ & -0.01 & 0.970 \\
\hline Docosapentaenoic C22:5 n-3 & $0.59(0.41)$ & -0.48 & $0.016^{*}$ & $0.55(0.38)$ & -0.43 & 0.126 \\
\hline DHA C22:6 n-3 & $6.67(1.33)$ & -0.66 & $<0.001^{*}$ & $5.93(0.85)$ & -0.66 & 0.011 * \\
\hline AA/DHA (wt \% ratio) & $2.65(0.59)$ & 0.66 & $<0.001^{*}$ & $2.85(0.58)$ & 0.68 & $0.007^{*}$ \\
\hline $\log _{10} T T$ & \multicolumn{3}{|c|}{$0.39(0.12$ to 1.04$)$} & \multicolumn{3}{|c|}{$0.41 \quad(-0.24$ to 0.80$)$} \\
\hline
\end{tabular}

Erythrocyte fatty acid concentrations (as weight \% of total fatty acids) are given as mean (SD) and log transit times (in seconds) as medians (ranges) * Statistically significant Spearman rank correlations $(r)$ between individual erythrocyte fatty acid concentrations and transit times. AA, Arachidonic acid; DHA, docosahexaenoic acid. 


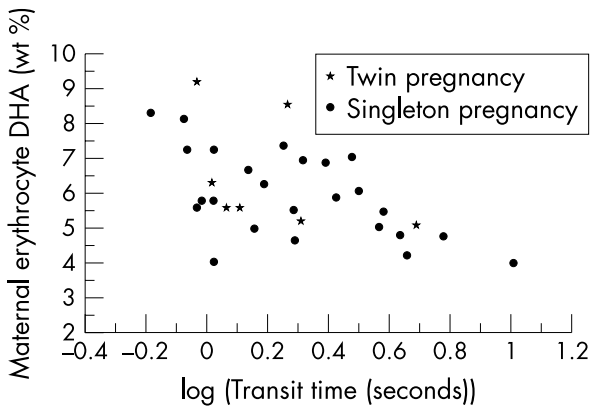

Figure 1 Distribution of maternal erythrocyte membrane total phospholipid docosahexaenoic acid (DHA; C22:6 n-3) concentrations (as weight \% of total fatty acids) and erythrocyte transit times (log transformed) in normal singleton and twin pregnancies. Maternal blood was obtained within 24 hours before delivery. Note that erythrocyte transit times in seconds are measured by their passage, at constant pressure, through a membrane of $5 \mu \mathrm{m}$ diameter pore size.

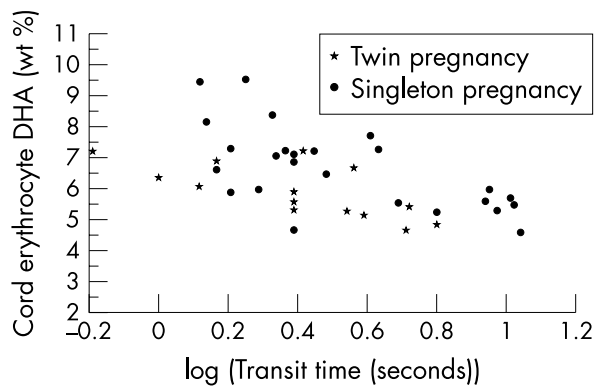

Figure 2 Distribution of umbilical cord blood erythrocyte membrane total phospholipid docosahexaenoic acid (DHA; C22:6 $\mathrm{n}-3$ ) concentrations (as weight $\%$ of total fatty acids) and erythrocyte transit times (log transformed) in normal singleton and twin pregnancies. Cord blood was obtained at delivery. Note that erythrocyte transit times in seconds are measured by their passage, at constant pressure, through a membrane of $5 \mu \mathrm{m}$ diameter pore size.

singleton and twin deliveries by Spearman's rank correlation. The relations between individual membrane fatty acids concentrations were similarly investigated both within and between (maternal $v$ cord) groups.

Retrospective regression analysis was performed to estimate the influence of gestational age and birth weight on maternal and cord DHA and maternal AA concentrations.

\section{RESULTS}

All birth weights were above the 3rd centile for gestation in both singleton and twin groups. Although only twin births that were near or at-term (34-38 weeks gestation) were included, these were of shorter mean gestation $(p<0.001)$ and lower mean birth weight $(\mathrm{p}<0.001)$ than those of the singletons (table 1 ).

In maternal and cord erythrocytes, strong inverse correlations (Spearman rank) with transit times were found only for n-3 LCPUFAs, DHA and docosapentaenoic (DPA; C22:5 n-3), in both singleton and twin delivery groups (tables 2 and 3 ). In contrast, only maternal erythrocyte AA and the AA/DHA (wt $\%)$ ratios directly correlated with transit times. Dihomo- $\gamma$ linolenic acid (C20:3 n-6) was associated with either no or a modest non-significant increase in deformabilities.

Within the n-3 fatty acids, maternal erythrocyte DHA correlated strongly with DPA in both singleton $(r=0.68$, $\mathrm{p}<0.001)$ and twin $(r=0.86, \mathrm{p}=0.012)$ pregnancies. A similar pattern was found for cord erythrocyte DHA and DPA (singletons: $r=0.72, \mathrm{p}<0.001 ;$ twins: $r=0.73, \mathrm{p}=0.005$ ).

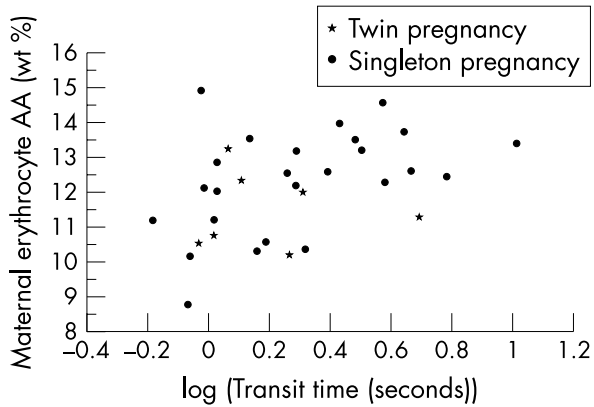

Figure 3 Distribution of maternal erythrocyte membrane total phospholipid arachidonic acid (AA; $20: 4 \mathrm{n}-6$ ) concentrations (as weight $\%$ of total fatty acids) and erythrocyte transit times (log transformed) in normal singleton and twin pregnancies. Maternal blood was obtained within 24 hours before delivery. Note that erythrocyte transit times in seconds are measured by their passage, at constant pressure, through a membrane of $5 \mu \mathrm{m}$ diameter pore size.

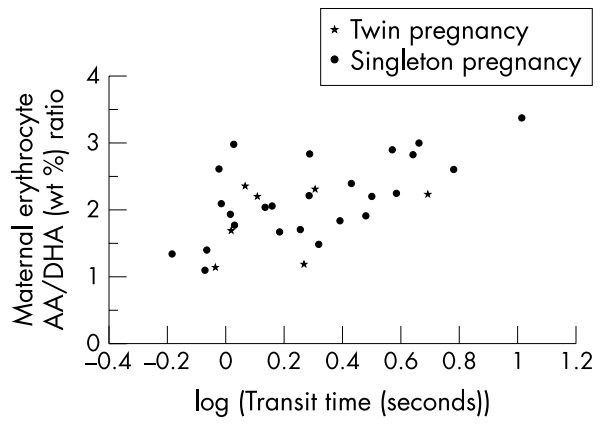

Figure 4 Distribution of maternal erythrocyte membrane total phospholipid arachidonic acid (AA; C20:4 n-6)/docosahexaenoic acid (DHA; $C 22: 6 n-3$ ) ratios ( $w$ t \%) and erythrocyte transit times (log transformed) in normal singleton and twin pregnancies. Maternal blood was obtained within 24 hours before delivery. Note that erythrocyte transit times in seconds are measured by their passage, at constant pressure, through a membrane of $5 \mu \mathrm{m}$ diameter pore size.

In singleton pregnancies only, a comparison of fatty acid concentrations between tissues yielded significant associations of maternal erythrocyte DHA with both cord DHA $(r=0.84$, $\mathrm{p}<0.001)$ and DPA $(\mathrm{r}=0.73, \mathrm{p}<0.001)$ and similarly of maternal erythrocyte DPA with cord DHA $(r=0.70$, $p<0.001)$. No significant correlations were found within the n-6 LCPUFAs nor between the major n-3 and n-6 fatty acids in maternal or cord erythrocytes from either singleton or twin pregnancies.

There was no significant contribution from gestational age (between 34 and 41 weeks gestation) to maternal DHA $\left(r^{2}=0.012\right)$, AA $\left(r^{2}=0.003\right)$ or cord DHA $\left(r^{2}=0.045\right)$. Similarly no significant influence of birth weight on maternal DHA $\left(r^{2}=0.008\right)$, AA $\left(r^{2}=0.000\right)$, or cord DHA $\left(r^{2}=0.070\right)$ was found.

No between group differences (singleton versus twin) in individual fatty acids were detected in maternal blood ( $t$ test); however, mean cord docosatetraenoic (C22:4 n-6; 3.56\% $v$ $2.96 \%)$ concentrations were significantly higher $(p=0.034)$ in the singleton group, whereas, although mean cord DHA concentration was higher, the difference was not significant $(\mathrm{p}=0.070)$.

Figures 1-4 show distributions of maternal and cord erythrocyte DHA and maternal AA concentrations and AA/DHA (wt \%) ratios with respect to transit times. Replacement by AA of its essential fatty acid precursor, linoleic, was the most prominent feature distinguishing cord from maternal erythrocyte fatty acid profiles in both pregnancy groups. 


\section{DISCUSSION}

Although we acknowledge that the complexity of human vasculature, particularly in pregnancy, cannot ever be adequately replicated in vitro, nevertheless, in this study we believe that we have shown a strong direct correlation between maternal and fetal (cord) erythrocyte deformabilities, as measured by in vitro filterability, and membrane n-3 LCPUFA content of DHA and DPA and a lesser inverse correlation with AA in the maternal circulation. We found that significant correlations in membrane LCPUFA concentrations were confined to the $n-3$ series. Absence of associations within the n- 6 series and between n-3 and n-6 LCPUFAs may have arisen because of our estimation of membrane total phospholipid fatty acids. By combining the analysis of phospholipids rich in LCPUFAs (phosphatidylethanolamine and phosphatidylserine) with those low in (phosphatidylcholine) or devoid of (sphingomyelins and cholesterol) LCPUFAs, a reduction in assay sensitivity may have arisen. This factor may warrant a larger study involving analysis of individual membrane phospholipid fatty acids. In addition, in this work, mean maternal and cord DHA concentrations are also higher than previously reported, even in maternal populations considered to have a diet high in DHA from marine sources. ${ }^{14}$ Maternal DHA concentrations above about $7 \%$ were associated with the virtual elimination of resistance to ultrafiltration of erythrocytes (fig 1), and, in the maternal circulation, correlated invariably with significant ( $>0.5 \%$ of total fatty acids) membrane incorporation (data not shown) of eicosapentaenoic acid (C20:5 n-3), which is indicative of fish oil consumption. A concurrent anonymised trial involving maternal fish oil intake may have influenced these findings in some pregnancies studied.

It may be that, after completion of cell differentiation and division, the mature enucleated and mitochondria free (using glucose as fuel) erythrocyte is not hindered by the genetic constraint imposed on actively replicating cells. Erythrocyte membrane incorporation of DHA and possibly AA may then be dictated more by diet than metabolic processes. In this environment a wide range of maternal erythrocyte AA/DHA (wt \%) ratios was encountered, with lower ratios, tending towards unity, associated with higher membrane deformabilities (fig 4).

Prenatally, the relatively high cord erythrocyte LCPUFA (DHA and AA) concentrations are attained through placental supply (at the expense of their essential fatty acid precursors) and illustrate their "biomagnification" in maternal to fetal transfer. ${ }^{15}$ Postnatally they are not sustained in the infant circulation, being replaced by their precursors, DPA and linoleic acid respectively.

As increased erythrocyte LCPUFA content is restricted to pregnancy, presumably some benefit must accrue to either the fetus or mother or both. Opinion is divided over what may represent appropriate membrane n-3 LCPUFA concentrations, the contentious issue being that increasing membrane incorporation of n-3 LCPUFA can be accompanied by a concomitant reduction in n-6 LCPUFA, including dihomo- $\gamma$-linolenic acid and AA, both important as parent molecules to vasoactive eicosanoid thromboxanes, leukotrienes, and prostaglandins. In this study AA concentrations significantly inversely correlated with maternal erythrocyte deformabilities in singleton pregnancies. The structural characteristics of AA (carbon chain length plus degree and position of unsaturation) would, in isolation, mitigate against such a possibility and may imply that opposing forces, probably involving eicosanoid function, are operating. This may be corroborated by the fact that dihomo- $\gamma$ linolenic acid, which is the precursor of the weakly active series 1 eicosanoids, had either a neutral or moderately positive effect on membrane flexibility. In addition, AA performs unique intracellular messenger functions dependent on its specific association with membrane phosphatidylinositol, which may influence its overall effect on membrane rheology. If greater incorporation of DHA is advantageous and does not jeopardise the function of n-6 LCPUFAs and their metabolites, then perhaps an increased maternal dietary intake of DHA should be recommended during pregnancy.

Consistently positive clinical outcomes from variations in membrane fatty acid compositions during pregnancy have not been documented because such research has been very limited. One study showed a strong inverse correlation of maternal erythrocyte DHA and DPA concentrations with the incidence of pre-eclampsia and a direct correlation with AA concentrations, ${ }^{16}$ and another produced negative results. ${ }^{17}$ Positive findings associated with high dietary fish oil intake in pregnancy have included the prolonging of gestational age at delivery, ${ }^{18}$ probably resulting from the increase in the ratio of the mildly vasoconstrictive thromboxane $\mathrm{A}_{3}$ synthesised from eicosapentaenoic acid to the more potent thromboxane $A_{2}$ from AA.

In general, in spite of the dearth of appropriately controlled trials, the earlier in pregnancy dietary intervention has been invoked, the more significant have been the outcomes. Introduction of a high fish diet to one group of mothers at 20 weeks gestation reduced the occurrence of pre-eclampsia and preterm delivery relative to the placebo group ${ }^{19}$; however, this was not replicated in a more recent early intervention (16-24 weeks gestation) study, which failed to show any effect of fish oil on pre-existing pre-eclampsia and the authors made no comment on preventive effects. ${ }^{20}$ Mean DHA concentration was lower in cord than corresponding maternal erythrocytes in our twin delivery group, a reversal of that found in singletons (tables 2 and 3 ). Although we recognise that twin pregnancies are at a higher risk of complications such as intrauterine growth restriction and pregnancy induced hypertension, no link with dietary deficiency has ever been established. A possible caveat to this may reside in those mothers with hepatic dysfunction in whom synthesis of LCPUFAs, especially DHA, may be compromised. It is suspected that human milk DHA content in the normal population is declining, probably because of maternal diet, and has reached mean concentrations ( $<0.2 \%$ of total fatty acids) that appear "inadequate" for the developing infant. ${ }^{21}$

If increasing erythrocyte n-3 LCPUFA concentrations by altering maternal diets is beneficial to vascular function, it should also be effective in providing the developing fetus with increased reserves of DHA, which at delivery, would be immediately available through breast feeding to the rapidly growing cardiovascular and neurological systems and possibly prevent the DHA deficiencies associated with formula feeding. ${ }^{22}$ As most human cell membranes are highly adaptable in incorporating fatty acids, it is difficult to comprehend why they contain such relatively high concentrations of DHA when preformed sources are virtually confined to the marine environment. This adaptability and specificity, as evidence now appears to show, may have enabled modern humans to develop relatively recently in close association with aquatic rather than terrestrial surroundings.

\section{ACKNOWLEDGEMENTS}

We thank the medical and midwifery staff of the Queen Mother's and Rutherglen maternity hospitals for their assistance in specimen collection, Drs Stein Bjornsson, Tracey Johnston, Alan Cameron, Barbara Holland, and Thomas Turner for their advice and encouragement, Eleanor Caine and Gareth Jones for their technical support, and all the mothers who participated in this project.

\section{Authors' affiliations}

M McFadyen, F Cockburn, University Department of Child Health, Royal Hospital for Sick Children and Queen Mother's Hospital, Yorkhill NHS Trust, Glasgow G3 8SJ, Scotland, UK

J Farquharson, Department of Biochemistry, Royal Hospital for Sick Children and Queen Mother's Hospital 


\section{REFERENCES}

1 British Nutrition Foundation's task force. Unsaturated fatty acids and early development. London: Chapman \& Hall, 1992:63-7.

2 FAO/WHO. The role of dietary fats and oils in human nutrition. Rome: $\mathrm{FAO}, 1978$.

3 Foreman-van Drongelen MM, Zeijdner EE, van Houwelingen AC, et al. Essential fatty acid status measured in umbilical vessel walls of infants born after a multiple pregnancy. Early Hum Dev 1996:46:205-15.

4 Pagnelli F, Maixent JM, Duran M, et al. Altered erythrocyte n-3 fatty acids in Mediterranean patients with coronary artery disease. Int J Cardiol 2001;78:27-32.

5 Ros HS, Cnattingius S, Lipworth L. Comparison of risk factors for preeclampsia and gestational hypertension in a population based cohort study. Am J Epidemiol 1998;147:1062-70.

6 Tzoumaka-Bakoula C, Lekea-Karanika V, Golding J, Thomas P. Hypertensive disorders of pregnancy in Greece. Eur J Obstet Gynecol Reprod Biol 1989;31:127-31.

7 Rossi E, Costa M. Fish oil derivatives as a prophylaxis of recurrent miscarriage associated with antiphospholipid antibodies (APL): a pilot study. Lupus 1993;2:319-23.

8 Balasch J, Carmona F, Lopez-Soto A, et al. Low-dose aspirin for prevention of pregnancy losses in women with primary antiphospholipid syndrome. Hum Reprod 1993:8:2234-9.

9 Farquharson J, Cockburn F, Patrick WA, et al. Infant cerebral cortex phospholipid fatty acid composition and diet. Lancet 1992;340:810-13.

10 Makrides M, Neumann MA, Byard RW, et al. Fatty acid composition of brain, retina and erythrocytes in breast- and formula-fed infants. Am J Clin Nutr 1994:60:19-94.

11 Cartwright IJ, Pockley AG, Galloway JH, et al. The effects of dietary omega-3 polyunsaturated fatty acids on erythrocyte membrane phospholipids, erythrocyte deformabilities and blood viscosity in healthy volunteers. Atherosclerosis 1985;55:267-81.
12 Jones JG, Holland BM, Humphrys J, et al. The flow of blood cell suspensions through 3 micron and 5 micron nucleopore membranes: a comparison of kinetic analysis with scanning electron microscope examination. Br J Haematol 1985;59:541-6.

13 Jamieson EC, Farquharson J, Logan RW, et al. Infant cerebellar gray and white matter fatty acids in relation to age and diet. Lipids 1999;34:1065-71.

14 Olsen SF, Hansen HS, Sommer S, et al. Gestational age in relation to marine $\mathrm{n}-3$ fatty acids in maternal erythrocytes: a study of women in the Faroe Islands and Denmark. Am J Obstet Gynecol 1991;164:1203-9.

15 Crawford MA, Hassam AG, Williams G, et al. Essential fatty acids and fetal brain growth. Lancet 1976;i:452-3.

16 Williams MA, Zingheim RW, King IB, et al. Omega-3 fatty acids in maternal erythrocytes and risk of preeclampsia. Epidemiology $1995 ; 6: 232-7$

17 Wang YP, Kay HH, Killam AP. Decreased levels of polyunsaturated fatty acids in preeclampsia. Am J Obstet Gynecol 1991;164:812-18.

18 Olsen SF, Sorensen JD, Secher NJ, et al. Randomised controlled trial of effect of fish oil supplementation on pregnancy duration. Lancet 1992;339:1003-7.

19 The People's League of Health. The nutrition of expectant and nursing mothers in relation to maternal and infant mortality and morbidity. Journal of Obstetrics and Gynaecology and the British Empire 1946:53:498-509.

20 Olsen SF, Secher NJ, Tabor A, et al. Randomised clinical trials of fish oil supplementation in high risk pregnancies. Fish Oil Trials In Pregnancy (FOTIP) Team. Br J Obstet Gynecol 2000;107:382-95.

21 Innis SM. Human milk and formula fatty acids. J Pediatr 1992;120:S566-1.

22 Farquharson J, Jamieson EC, Abbasi KA, et al. Effect of diet on the fatty acid composition of the major phospholipds of infant cerebral cortex. Arch Dis Child 1995;72:198-203.

\section{This month in the Archives of Disease in Childhood}

The following papers appearing in the March 2003 issue of $A D C$ may be of interest to readers of Fetal and Neonatal.

Congenital adrenal hypoplasia presenting as a chronic repiratory condition. K Schwartz, $R$ Thwaites, A Mindford, et al

Breast feeding and respiratory morbidity in infancy: a birth cohort study. W H Oddy, P D Sly, N H de Klerk, et al

Long term follow up of serostatus after maternofetal parvovirus B19 infection. J Dembinski, A M Eis-Hübinger, J Maar, et al

Hib vaccination in infants born prematurely. P T Heath, R Booy, J McVernon, et al

The British Association of Perinatal Medicine: the first 25 years (1976-2000). P M Dunn 